\title{
An Integrated Airport Operations Interface Integrating and Automating Airport Operations
}

\author{
Zhexing Yan \\ School of Civil and Building Engineering \\ Loughborough University \\ Loughborough, Leicestershire, UK \\ z.yan@lboro.ac.uk
}

\author{
Dr. Mahroo Eftekhari \\ School of Civil and Building Engineering \\ Loughborough University \\ Loughborough, Leicestershire, UK \\ m.m.eftekhari@lboro.ac.uk
}

\begin{abstract}
Integrating and Automating Airport Operations, the main driver for this research is to decrease any detrimental effects of airport operations upon the environment. It represents a wide ranging multi-disciplinary and crossinstitutional initiative to exploit recent research advances in automated search methodologies and decision support techniques for air operations (and other related areas). This project will open up a range of exciting and ambitious research directions in the crucially important area of airport operations. This research will build integrated computational models of three key airport operations: Runway Sequencing, Ground Movement, and Gate Assignment. The Human Machine Interface (HMI) is based on the operations making a platform to link the three key areas together, to create an integrated user interface. This paper addressed some of the basic knowledge in applying Level CheckingSystem combined with Case-based Reasoning (CBR) to improve in the Decision Support System developing. The paper also demonstrates the early stage of HMI design process.
\end{abstract}

Keywords-Airport operations; Human machine interface; User interface; Decision support system

\section{INTRODUCTION}

In the airfield, there has been significant previous research into the stand/gate allocation problem, the arrival and departure sequencing problems, the ground movement and stand operations problems. However, most research has considered only one of these problems at a time. This project considers the ways in which these problems are linked, and the ways in which previous academic models have deviated from the real problems, in order to increase the level of automation which is practical for real airports.

One of the problems of a holistic airport management system would be the understand ability of the solutions or schedules. Different operators usually focus on individual aspects of the problem (e.g. gate assignment [1], only arrival sequencing, etc.). In an integrated system, one might have to make locally undesirable solutions (e.g. accept a delay in arrivals to facilitate runway crossings, move an aircraft to an inconvenient gate to facilitate ground movement or prevent contention). A local or myopic system user would, therefore, be surprised on why they have to take a hit, or why some part of a solution is obviously undesirable. From this perspective, a HMI with explanatory power could be an interesting direction e.g., not only displaying the solution, but also stating the reason for the solution. What-if analysis, rule based systems, or "reasoning" methods could potentially help to deduce such information from the solutions.

\section{INTEGRATING AND AUTOMATING AIRPORT OPERATIONS GROUP}

The Integrating and Automating Airport Operations group is a multi-disciplinary project involving three Universities.University of Nottingham, who is investigating three key problem areas (Runway Sequencing, Ground Movement and Gate Assignment); University of Lincoln focuses on the fuel consumption; the task of LoughboroughUniversityis to link the three key areas together based on the operations making a platform, to create an integrated user interface. The Group focuses on the Manchester Airport, developing the operations particularly based on the data from Manchester Airport.The group works closely with colleagues fromairport to ensure continuous access to user specification and data.

\section{DECISION SUPPORT SYSTEM DEVELOPING}

\section{A. Human Machine Interface Developing Process}

The HMI developing process begins with defining the users and usability requirements. The main aim of the system is to model user task and their objectives. User interface (UI) design provides graphical view for user.

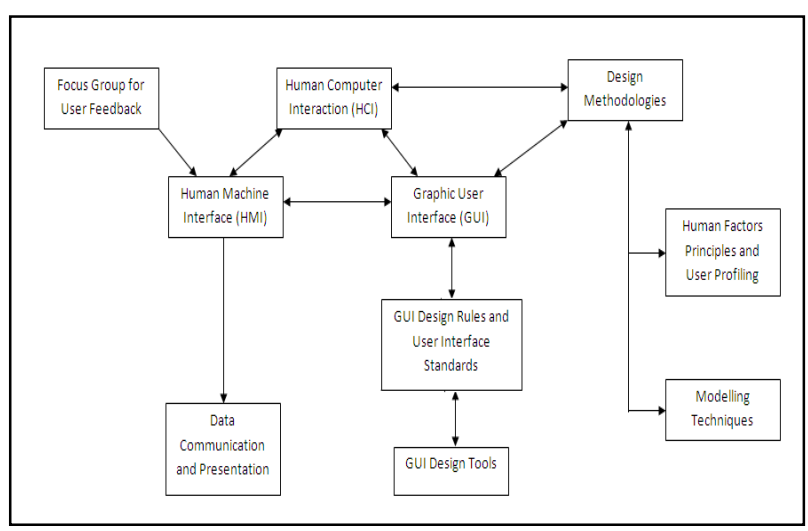

Figure 1. HMI Development. 
The overview diagram shown in Figure 1 is an idealized representation of the processes and how they related to each other.

While a schematic overview diagram helps with communication, it is important also to acknowledge its limitations. It is recognized that the real-world projects are almost always more complex [2].

\section{B. Case-Based Reasoning for Decision Support System}

Having access to relevant case history in problem solving reduces the need for problem analysis because solution chunks from old problems can be reused and less in-depth analysis of the new problem is required. The conventional wisdom is that Case-Based Reasoning (CBR) is particularly appropriate in weak theory domains where the important influences and interactions are not well understood. The objective is that the problem of modeling these interactions can be finessed by reusing previously solved problems stored as cases $[3,4]$.

The aim of this research is to develop a Multi-system as shown in Figure 2, based on a decision support center combining a CBR system [5].The reason for creating an integrated HMI is not only to display the solutions from a decision support system, but also be able to analysis the reason for the decision. This kind of HMI would be used in education and training purpose as well.

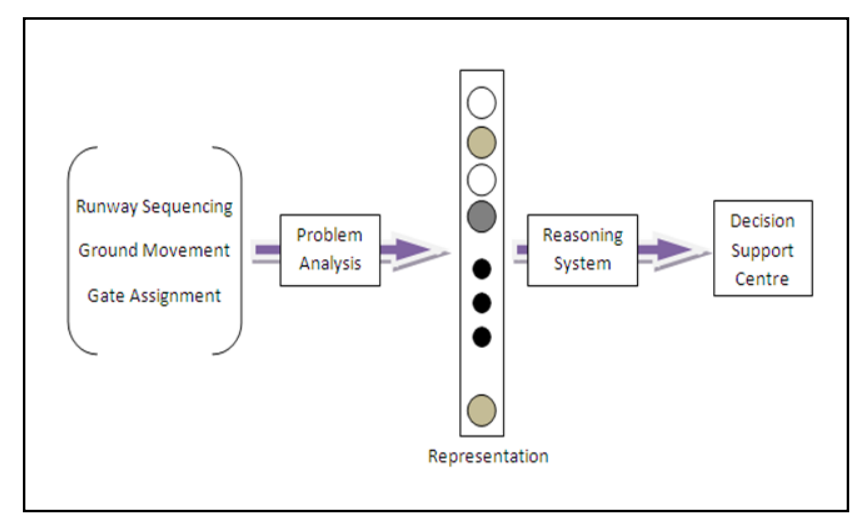

Figure 2. Development of a Multi-system.

As the Multi-system diagram (Figure 2) shows, the real world problems are analyzed and are appropriately represented to be analyzed by a reasoning system, then a decision support center will provide an integrated solution.

\section{Level Checking System}

- Level 1 Quick Check

Recently display the model data and model date in the interface, do the color mapping and analysis. Analyse and minimize the variables which users can change and play around in the interface, the results of the basic human interaction will be checked through Quick Check first. The Quick Check bans the obvious faults (e.g. opposite stands push back at the same time, or near stands use the taxi way at the same time etc.), and sends the faults to Reason Advisor, which is based on the reasoning system to display the fault reasons to users. The other changes will be submitted to the next level check (Figure 3).

- $\quad$ Level 2 Long Check

In this level, the changes will be submitted to optimize system. The gate model and runway model do the long check and give the feedback, analyze the influence of the reschedule, which shows how good or bad if do so. The Reason Advisor collects the bad user changes, and gives the reasons to users about the faults. Other useful analysis will be passed to the next level (Figure 3).

- Level 3 Debug

According to the previous two levels checks, users would be able to freeze the reasonable changes and fix the rest (Figure 3).

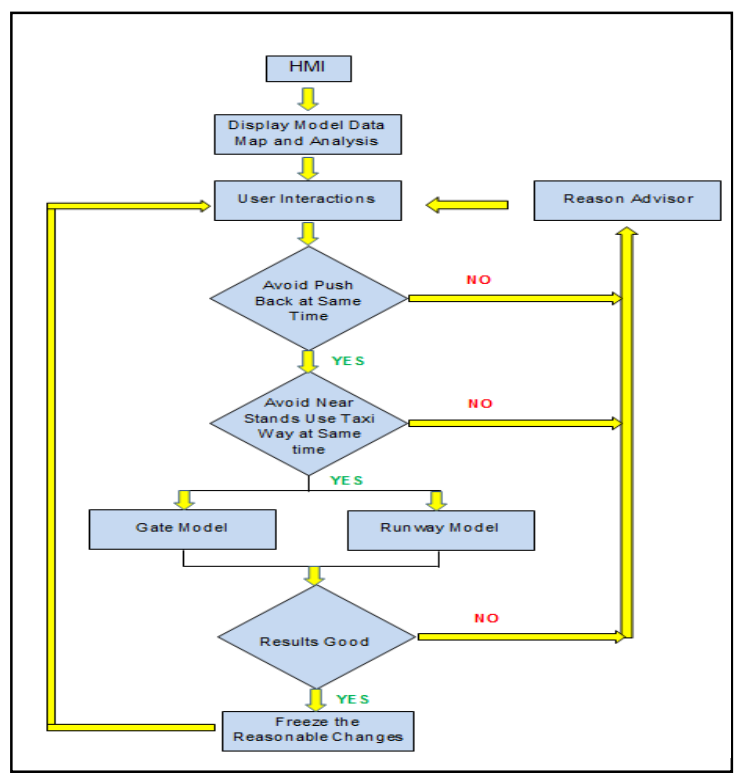

Figure 3. Level Checking diagram.

\section{HuMAN MACHINE INTERFACE DESIGN}

As this group project is divided into several areas, (Runway Sequencing, Ground Movement and Gate Assignment), a User Interface is needed to integrate the information and automate the operations.The User Interface Design optimizes coordination and integrates the full information from each individual area.

\section{A. Essential User Interface Elements}

Before the UI design, according to the operational information analysis, all the data can be allocated in different scenarios, and each scenario has several elements which are related closely to the real operations. Figure 4shows the essential UI elements in the initial design of the basic UI layout. 


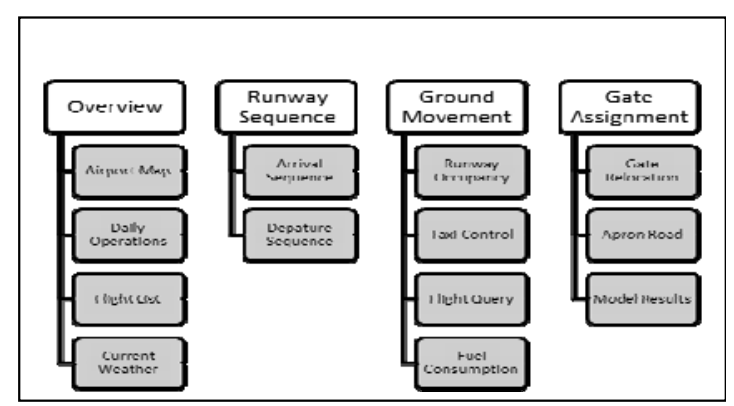

Figure 4. User Interface Elements.

\section{B. Graphical User Interface (GUI) Design}

To create a useful and easy learning UI, the UI layout becomes a key factor for users to learn and control, so that before programming the data base, a better UI layout needs to be decided.

A part of layout design idea is from the literature review of existing UI, e.g. SensisAerobahn [6] Interface designs several icons for integrating and visible graphic for reporting, which are convenient for user to operate and monitor.

In the initial design stage, only Overview and Gate UI layouts have been set up. The following four figures show the element panels design.

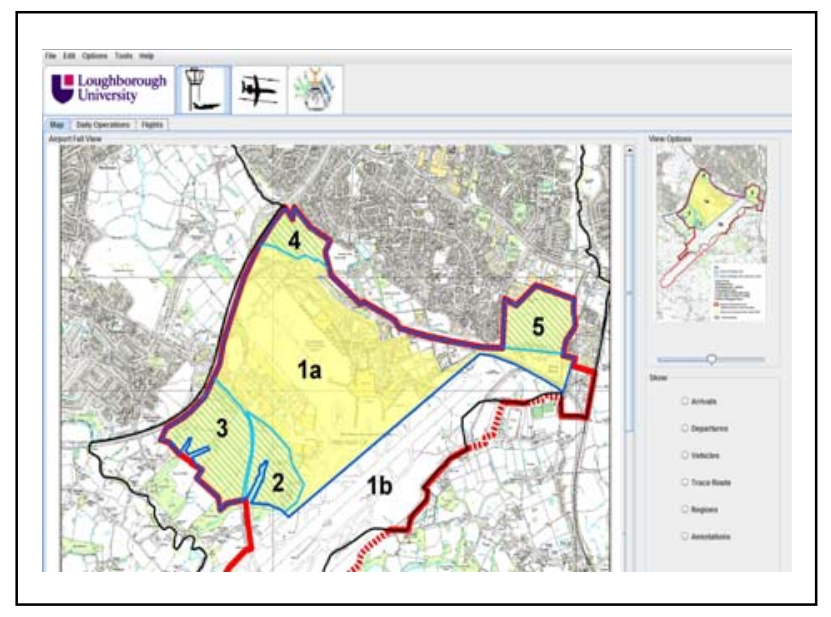

Figure 5. Airport Map panel.

Airport Map panel (Figure 5) gives the graphical overview of the airport and flights situation. The airport map is shown in the Airport Full View label, however, only part of the full map is in the visible area, so that users can control the scroll bar and adapt the View Options by zooming in and out. Furthermore, the selection items in the Show Label are for users tochoose what kinds of information to show on the map.

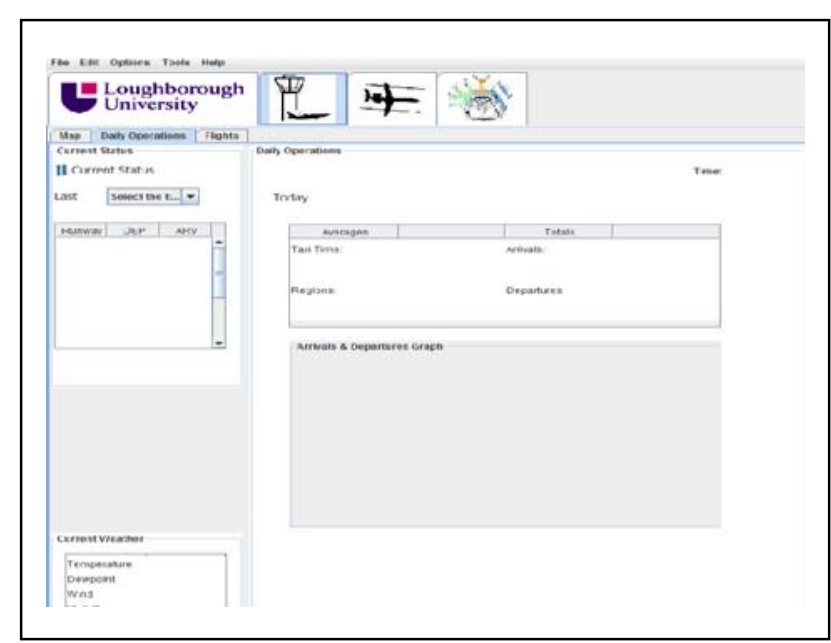

Figure 6. Daily Operations panel.

Daily Operations panel (Figure 6) mainly displays the daily arrivals and departures by selecting time and date. According to the operation data, the UI will automatically create appropriate graph for reporting purpose. Current Weather data as a reference factor appears on the left corner of the UI.

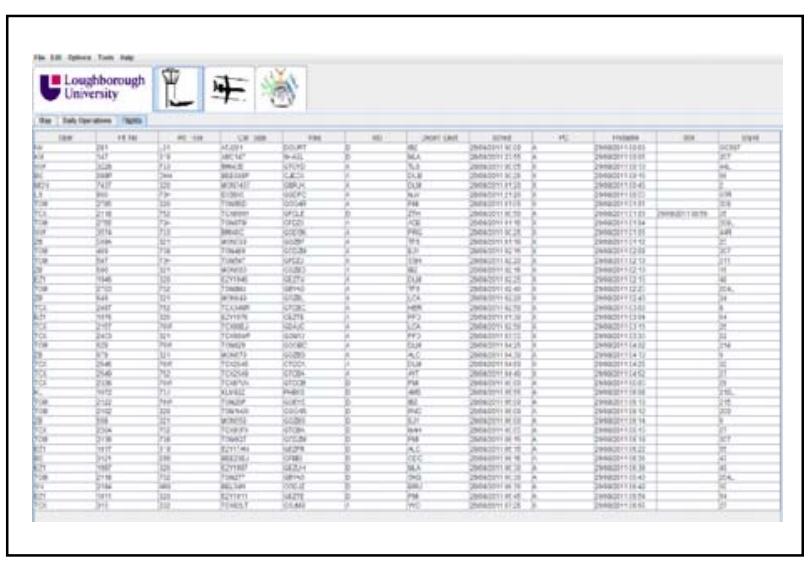

Figure 7. T2 Real Time Data $29^{\text {th }}$ August 2011 in HMI Flights panel.

Flight panel (Figure 7) displays the basic air flight information (e.g. departure time, arrival time, runway, gate etc.) in the main table, once clicking on each flight; the detailed information will appear in the Flight Info label. In addition, the Passengers Info plans to send numbers and details of passengers to the operation and security; this part is set in the layout, but if not possible to get passengers information from airport system, it will be rebuilt in the future.

Gate Assignment has the closely link with the Ground Movement. To do the gate allocation need the data from Runway Sequencing and Ground Movement before the analysis. After gate relocation, the results are displayed on apron road and model results panel. 


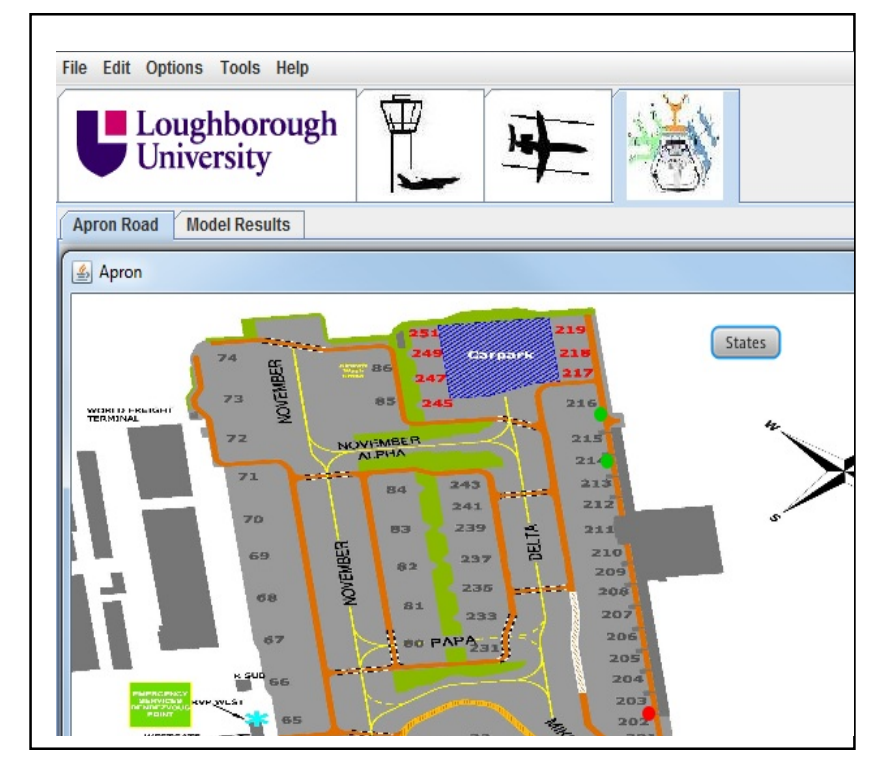

Figure 8. Apron Road panel.

Apron Road panel (Figure 8) as a part of the gate allocation control scenario, gives the gate situations information to users. Figure 8 demonstrates Manchester Airport T2 gates. The red points in the map mean flights arrive at those stands, and the green points show flights departure from those stands; as the time pass, the occupancies of each gate will be automatically updated by time.

\section{Data Sharing}

Java programming language has been used as the basic language tool for the whole group to achieve the goal of the project. Recently, the data sharing between the group members via CSV (comma-separated values) files, by saving the simulation results and programming code as CSV files, and inputting the CSV data files to the HMI scenario to display the results.

\section{Gate Allocation Analysis}

In order to make a case study of Manchester Airport gate allocation, terminal two (T2) has been selected for an analysis. As the most distant terminal from the runways, T2 has fourteen gates with air bridges, numbered from 202 to 215. It is now the quietest terminal but the airport tries to revive it by encouraging airlines to use it more often.

The real time operation data have been downloaded from the date $29^{\text {th }}$ August 2011 to $4^{\text {th }}$ September 2011 by the airport operator. The real time data have been transferred to HMI Flights information panel (Figure 7) as reference data.

According to the one week real data, the analysis focuses on one ofthe busy summer day $29^{\text {th }}$ August 2011. Reorganized the real data, filtered the same flight by the registration number, the results pointed out that there were 41 different flights arrival and departure at T2.
The arrival time and departure time have been displayed by minutes, for convenient comparing purpose. T2 Arrivals \& Departures $29^{\text {th }}$ August 2011 timeline chart application (Figure 10) bases on the real time and model one day data to display the daily $\mathrm{T} 2$ gate states.

In Figure 10, the $\mathrm{X}$ axis is time in minutes; the $\mathrm{Y}$ axis is the gate number of $\mathrm{T} 2$. In real time analysis, arrivals have been presented as the yellow points, and the departures have been presented as the blue points; in the model analysis, arrivals have been presented as the red points, and the departures have been presented as the green points. It is easy to identify that in the real time performance, gate 207 to 211 were quite busier than others. Figure 9 shows the comparison of fight number of two kinds of data in each gate. In the real time data, four gates were not used, two gates held 5 flights a day, four gates held 4 flights a day, and others had no more than 3 flights. However, in the model results, all the gates were used and no more than 3 flights a day.

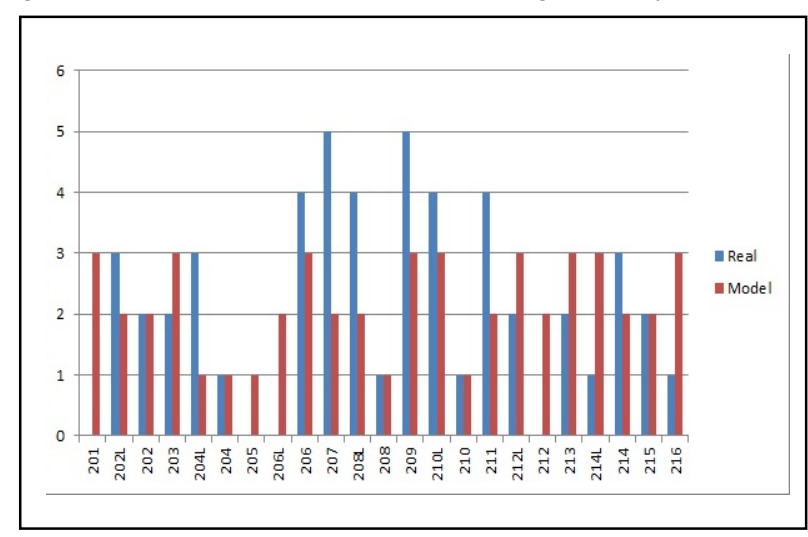

Figure 9. Comparison of Real and Model Flight Number $29^{\text {th }}$ August.

The analysis shows the crowded area of T2, the problem could be solved by integrating ground and runway information. Meanwhile, the time lags between the arrivals and departures, and also between different flights scheduled in the same gate would possibly be relocated.

\section{ACKNOWLEDGMENT}

The authors would like to acknowledge the funding from EPSRC and support from Manchester airport, Nottingham and Lincoln Universities.

\section{REFERENCES}

[1] Y. Chen, A rule-based reactive model for the simulation of aircraft on airport gates, Knowledge-Based Systems 10, 1998, pp. 225-236.

[2] D. Redmond-Pyle and A. Moore, Graphical User Interface Design and Evaluation, Prentice Hall International, 1995.

[3] P. Cunningham and A. Bonzano, Knowledge engineering issues in developing a case-based reasoningapplication, Knowledge-Based Systems 12, 1999, pp. 371-379.

[4] M. Brandena, N. Wiratungaa, D Burtonb and S. Crawa, Integrating case-based reasoning with an electronic patient record system, Artificial Intelligence in Medicine 51, 2011, pp. 117-123.

[5] A. Bonzano, P. Cunningham and C. Meckiff, ISAC: A CBR system fordecision support in air traffic control, advances in case-based reasoning,in: I. Smith, B. Faltings (Eds.), Proceedings of the 1996 


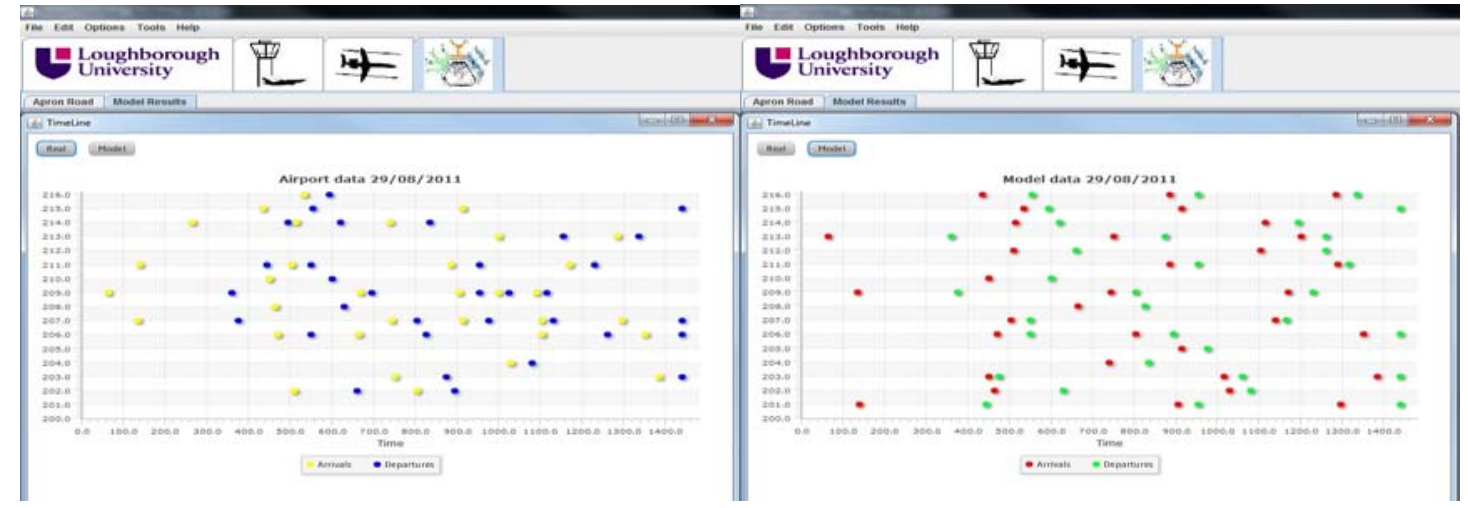

Figure 10. T2 Arrivals \& Departures $29^{\text {th }}$ August 2011 timeline chart. 